Published as APJL, 836, L1

Preprint typeset using $\mathrm{LT}_{\mathrm{E}} \mathrm{X}$ style emulateapj v. 04/20/08

\title{
ON THE ACCRETION RATES AND RADIATIVE EFFICIENCIES OF THE HIGHEST REDSHIFT QUASARS
}

\author{
BenNy Trakhtenbrot ${ }^{1,4}$, MARTA VOLONTERI ${ }^{2}$, AND PRIYAMVAdA NATARAJAN ${ }^{3}$ \\ Published as ApJL, 836, L1
}

\begin{abstract}
We estimate the accretion rates onto the supermassive black holes that power 20 of the highest-redshift quasars, at $z \gtrsim 5.8$, including the quasar with the highest redshift known to date - ULAS J1120 at $z=7.09$. The analysis is based on the observed (rest-frame) optical luminosities and reliable "virial" estimates of the BH masses of the quasars, and utilizes scaling relations derived from thin accretion disk theory. The mass accretion rates through the postulated disks cover a wide range, $\dot{M}_{\text {disk }} \simeq 4-190 M_{\odot} \mathrm{yr}^{-1}$, with most of the objects (80\%) having $\dot{M}_{\text {disk }} \simeq 10-65 M_{\odot} \mathrm{yr}^{-1}$, confirming the Eddington-limited nature of the accretion flows. By combining our estimates of $\dot{M}_{\text {disk }}$ with conservative, lower limits on the bolometric luminosities of the quasars, we investigate which alternative values of $\eta$ best account for all the available data. We find that the vast majority of quasars $(\sim 85 \%)$ can be explained with radiative efficiencies in the range $\eta \simeq 0.03-0.3$, with a median value close to the commonly assumed $\eta=0.1$. Within this range, we obtain conservative estimates of $\eta \gtrsim 0.14$ for ULAS J1120 and SDSS J0100 (at $z=6.3$ ), and of $\gtrsim 0.19$ for SDSS J1148 (at $z=6.41$; assuming their BH masses are accurate). The implied accretion timescales are generally in the range $t_{\mathrm{acc}} \equiv M_{\mathrm{BH}} / \dot{M}_{\mathrm{BH}} \simeq$ $0.1-1 \mathrm{Gyr}$, suggesting that most quasars could have had $\sim 1-10$ mass $e$-foldings since BH seed formation. Our analysis therefore demonstrates that the available luminosities and masses for the highest-redshift quasars can be explained self-consistently within the thin, radiatively efficient accretion disk paradigm. Episodes of radiatively inefficient, "super-critical" accretion may have occurred at significantly earlier epochs (i.e., $z \gtrsim 10$ ).

Subject headings: galaxies: active — galaxies: nuclei — quasars: general — black hole physics
\end{abstract}

\section{INTRODUCTION}

The existence of luminous quasars as early as $z \sim 6-7$ suggests that supermassive black holes (SMBHs) with masses of order $M_{\mathrm{BH}} \sim 10^{9} M_{\odot}$ were in place less than $1 \mathrm{Gyr}$ after the Big Bang. This is explicitly shown by observations that trace the gas dynamics in the close vicinity of the accreting $\mathrm{SMBHs}$ (Kurk et al. 2007; Willott et al. 2010; De Rosa et al. 2011; Trakhtenbrot et al. 2011; De Rosa et al. 2014). Such masses require continuous, exponential growth at the Eddington limit, $L / L_{\mathrm{Edd}}=1$, for almost the whole age of the universe at that time, and these conditions may not be necessarily ubiquitous among early SMBHs (e.g., Treister et al. 2013; Habouzit et al. 2016; Trakhtenbrot et al. 2016; Volonteri \& Reines 2016).

The ability of SMBHs to grow to $M_{\mathrm{BH}} \sim 10^{9} M_{\odot}$ depends, critically, on the radiative efficiency of the accretion flow defined as $\eta \equiv L_{\mathrm{bol}} / \dot{M}_{\mathrm{acc}} c^{2}$, where $\dot{M}_{\text {acc }}$ is the mass inflow rate and $L_{\text {bol }}$ is the emerging bolometric luminosity. Most calculations of early BH growth assume a universal value of $\eta \simeq 0.1$, relying on the ensemble properties of quasars and relic SMBHs across all cosmic epochs.

In reality, however, the role of $\eta$ in early $\mathrm{BH}$ growth is more complex. Individual systems should have various $\eta$, as indeed suggested by the observed range of BH spins (see, e.g., the review by Reynolds 2014 and also Davis \& Laor 2011; Trakhtenbrot 2014; Capellupo et al. 2016). The value of $\eta \simeq 0.1$ is within the range expected in optically thick, geometrically thin accretion disks, where $\eta \sim 0.04-0.32$, de-

\footnotetext{
Electronic address: benny.trakhtenbrot@phys.ethz.ch

${ }^{1}$ Institute for Astronomy, Department of Physics, ETH Zurich, WolfgangPauli-Strasse 27, CH-8093 Zurich, Switzerland

${ }^{2}$ Institut d'Astrophysique de Paris, UPMC et CNRS, UMR 7095, 98 bis bd Arago, F-75014 Paris, France

${ }^{3}$ Department of Astronomy, Yale University, 260 Whitney Avenue, New Haven, CT 06511, USA

${ }^{4}$ Zwicky postdoctoral fellow
}

pending on the BH spin. However, we recall that $\eta$ would be much lower for geometrically thick accretion disks, such as for advection-dominated flows, characteristic of significantly sub-Eddington accretion (e.g., Narayan \& Yi 1995), or "super-critical" accretion flows (e.g., Paczyńsky \& Wiita 1980). Additionally, in these regimes, the luminosity is not proportional to the accretion rate, but to the accretion rate squared and the logarithm of the accretion rate, respectively.

Importantly, the relevance of either low- $\eta$ mechanisms or the assumption of a universal $\eta=0.1$ to the observed population of the earliest known quasars, are not yet established.

In this Letter we use insights from thin accretion disk theory and basic observables of some of the highest-redshift quasars known to date, at $z \simeq 6$, to investigate the mass accretion rates and the corresponding radiative efficiencies powering these systems. This work assumes a cosmological model with $\Omega_{\Lambda}=$ $0.7, \Omega_{\mathrm{M}}=0.3$, and $H_{0}=70 \mathrm{~km} \mathrm{~s}^{-1} \mathrm{Mpc}^{-1}$.

\section{METHOD, SAMPLE, AND DATA}

The goal of the present study is to test whether the currently available data for the highest-redshift quasars known to date can be self-consistently explained within the thin accretion disk model and to estimate the corresponding accretion rates $\left(\dot{M}_{\text {disk }}\right)$ and radiative efficiencies $(\eta)$. The method we use is based on two fundamental assumptions: that the SMBHs we study are powered by thin accretion disks and that their masses are reliably known. In thin-disk accretion flows, the rest-frame optical continuum emission $\left(\lambda_{\text {rest }} \gtrsim 4500 \AA\right)$, originating primarily from the outer disk, follows a powerlaw form, $L_{v} \propto\left(M_{\mathrm{BH}} \dot{M}_{\mathrm{disk}}\right)^{2 / 3} v^{1 / 3}$ (see, e.g., Davis \& Laor 2011, and references therein). Rewriting $\dot{M}_{\text {disk }}$ in terms of $M_{\mathrm{BH}}$ and the (monochromatic) continuum luminosity along 
this power-law tail provides

$$
\dot{M}_{\mathrm{disk}} \simeq 2.4\left(\frac{\lambda L_{\lambda, 45}}{\cos i}\right)^{3 / 2}\left(\frac{\lambda}{5100 \AA}\right)^{2} M_{8}^{-1} M_{\odot} \mathrm{yr}^{-1}
$$

where $\lambda$ is the (rest-frame) wavelength at which the continuum is measured, $\lambda L_{\lambda, 45} \equiv \lambda L_{\lambda} / 10^{45} \mathrm{erg} \mathrm{s}^{-1}$ denotes the monochromatic luminosity, and $M_{8} \equiv M_{\mathrm{BH}} / 10^{8} M_{\odot}$. cos $i$ represents the inclination between the line of sight and the polar axis of the disk (here we adopt $\cos i=0.8$, as appropriate for broad-line quasars). The derivation of this expression is discussed in detail in, e.g., Davis \& Laor (2011) and Netzer $\&$ Trakhtenbrot (2014). This approach was used in several recent studies of accretion flows for samples of quasars to $z \simeq 3.5$ (e.g., Bian \& Zhao 2003; Davis \& Laor 2011; Wu et al. 2013; Trakhtenbrot 2014).

At $z \gtrsim 6$ the estimation of $\dot{M}_{\text {disk }}$ through Equation 1 necessitates flux measurements at $\sim 3.5-10 \mu \mathrm{m}$, and $K$-band spectroscopy of the $\mathrm{Mg}$ II $\lambda 2798$ broad emission line (for $M_{\mathrm{BH}}$ estimation; see, e.g., Trakhtenbrot \& Netzer 2012, hereafter TN12, and references therein). Here, we focus only on those $z \gtrsim 6$ quasars for which such data are publicly available.

Compiling all the $z \simeq 6$ quasars for which Mg II-based estimates of $M_{\mathrm{BH}}$ are available from NIR spectroscopy, we find 35 objects in the studies of Iwamuro et al. (2004), Jiang et al. (2007), Kurk et al. (2007), Kurk et al. (2009), Willott et al. (2010), Venemans et al. (2013), De Rosa et al. (2014), and Venemans et al. (2015). We additionally include the wellstudied $z \simeq 6.4$ quasar SDSS J1148 (from Barth et al. 2003), and the highest-redshift quasar known to date, ULAS J1120 $(z=7.085$; Mortlock et al. 2011) We finally include the extremely luminous and massive quasars $\mathrm{J} 0100+2802(z=6.3$; $\mathrm{Wu}$ et al. 2015) and J0306+1853 ( $z=5.363$; Wang et al. 2015). Although J0306 is at a lower redshift, we include it to test if the MIR-based high-redshift quasar selection methods may be unveiling a distinct population of SMBHs. Throughout this work, we highlight the results obtained for these four quasars of interest, but note here that they should be viewed as part of an ensemble of quasars.

For some of the quasars there are multiple published NIR spectra and/or Mg II profile measurements. Whenever possible, we have consistently used the detailed measurements performed by De Rosa et al. (2011). These replace the measurements provided by Iwamuro et al. (2004), Jiang et al. (2007), Kurk et al. (2007, except for J0836), and Kurk et al. (2009). For sources with multiple sets of measurements in De Rosa et al. (2011), we selected those with the smaller uncertainties on FWHM(Mg II). Using the measurements of $L_{3000}$ and FWHM(Mg II) reported in the selected studies (and adjusted for our chosen cosmological model), we re-calculated all $M_{\mathrm{BH}}$ estimates following the prescription of TN12 (see also Shen et al. 2011). These accurate estimates of $M_{\mathrm{BH}}$ are known to carry systematic uncertainties of up to $0.5 \mathrm{dex}$ (TN12 and references therein).

We then compiled rest-frame optical photometric data, obtained with the Spitzer and WISE IR space telescopes. For nine of the quasars, we use Spitzer/IRAC data reported in the studies of Jiang et al. (2006), Leipski et al. (2014), and Barnett et al. (2015, for ULAS J1120). Whenever possible, we used the Leipski et al. (2014) measurements for homogeneity. For 11 quasars we use WISE measurements in the W1 and W2 bands, obtained by cross-matching our sample with the AllWISE data release, within $5^{\prime \prime}$ of the optical coordinates

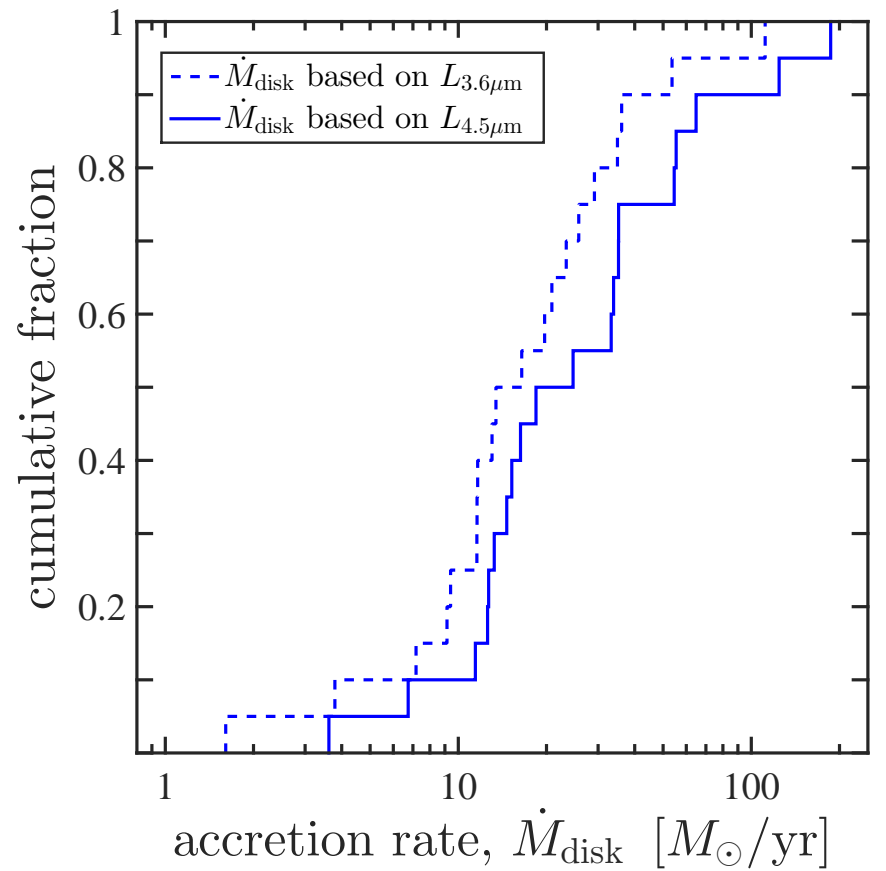

FIG. 1.- Cumulative distribution function of our estimates of accretion rates through the disks, $\dot{M}_{\text {disk }}$, based on Equation 1. The dashed and solid lines represent the estimates based on $L_{3.6}$ and $L_{4.5}$, respectively. The systematically higher $L_{4.5}$-based estimates of $\dot{M}_{\text {disk }}$ result in more conservative constraints on $\eta$ (i.e., lower values) and shorter accretion timescales.

of the sources. In all cases where both Spitzer and WISE measurements are available, we preferred the higher spatial resolution and sensitivity Spitzer data. The first two bands of the IRAC or WISE cameras have effective wavelengths of roughly 3.6 and $4.5 \mu \mathrm{m}$. For our sample's redshift range, these correspond to rest-frame wavelengths of about 48505340 and 6065-6675 $\AA$, respectively. We verified that none of our sources is affected by blending with neighboring WISE sources. All Spitzer and WISE measurements were converted to flux densities using standard procedures (i.e., Wright et al. 2010; Jarrett et al. 2011). Monochromatic luminosities were calculated assuming the $\mathrm{Mg}$ II-based redshifts reported in the aforementioned NIR studies (see Table 1).

Our final sample includes 20 quasars with reliable estimates of $M_{\mathrm{BH}}, 9$ with Spitzer data and the remaining 11 with WISE data. We verified that none of the choices we made in compiling the data set has significant effects on our results. The heterogeneous nature of our sample - drawn from several surveys of varying depth, and our obvious focus on vigorously accreting SMBHs at this extremely high redshift regime, mean that our sample is most probably not representative of the entire population of (active) SMBHs at $z \simeq 6$.

We calculated $\dot{M}_{\text {disk }}$ for the 20 quasars through Equation 1, using the (re-calculated) $\mathrm{Mg}$ II-based $M_{\mathrm{BH}}$ estimates, and the monochromatic luminosities observed in either of the IR bands (i.e., $\sim 3.6$ and $4.5 \mu \mathrm{m}$, hereafter $L_{3.6}$ and $L_{4.5}$, respectively). We list all the quantities relevant for the present analysis, including the chosen sources of all measurements, in Table 1 .

\section{RESULTS}

Figure 1 presents the derived accretion rates through the disks, $\dot{M}_{\text {disk }}$, for all the sources in our sample, and based on the two different (rest-frame) optical luminosities. The accre- 


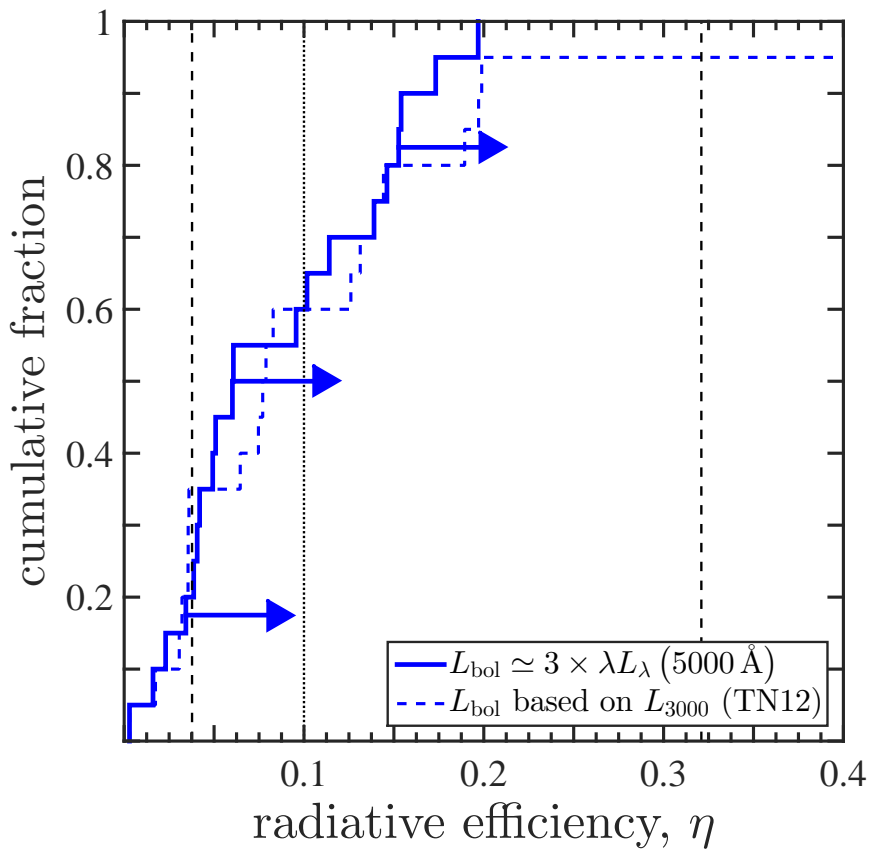

FIG. 2.- Cumulative distribution function of radiative efficiency estimates, $\eta$. The solid line traces the values obtained using the conservative assumption of $L_{\mathrm{bol}}=3 \times L_{3.6}$ (roughly $\left.L_{\mathrm{bol}} \simeq 3 \times L_{5100}\right)$ and the generally higher $L_{4.5^{-}}$ based estimates of $\dot{M}_{\text {disk }}$. The dashed line traces the $\eta$ estimates based on $L_{\text {bol }}\left(L_{3000}\right)$, using the TN12 bolometric corrections. The vertical dashed lines mark range of radiative efficiencies expected for thin accretion disks around spinning BHs $(0.038 \lesssim \eta \lesssim 0.32)$. The dotted vertical line marks $\eta=0.1$.

tion rates we obtain using $L_{4.5}$ are in the range $\dot{M}_{\text {disk }} \simeq 3.6-$ $187 M_{\odot} \mathrm{yr}^{-1}$, with 16 of the quasars $(80 \%)$ having $\dot{M}_{\text {disk }} \simeq$ $10-65 M_{\odot} \mathrm{yr}^{-1}$. We note that some of the variance in $\dot{M}_{\mathrm{BH}}$ in our sample may be attributed to the significant systematic uncertainties in $M_{\mathrm{BH}}$, and the form of Equation 1. Notwithstanding this limitation, we obtain $\dot{M}_{\text {disk }}=11.4 M_{\odot} \mathrm{yr}^{-1}$ for ULAS J1120, while for SDSS J1148 and SDSS J0100 we find $\dot{M}_{\text {disk }} \simeq 16.3$ and $54.6 M_{\odot} \mathrm{yr}^{-1}$, respectively. The accretion rate we find for SDSS J0306 is in excellent agreement with that of SDSS J0100, which is expected given the very similar masses and continuum luminosities of the two quasars (both within 0.1 dex). The $L_{3.6}$-based $\dot{M}_{\text {disk }}$ estimates are systematically lower than those based on $L_{4.5}$, by about 0.19 dex (median value). This is probably due to the fact that the $4.5 \mu \mathrm{m}$ band includes the strong broad $\mathrm{H} \alpha$ line emission, which is expected to be stronger by a factor of $\sim 3$ compared with the $\mathrm{H} \beta$ line, covered in the $3.6 \mu \mathrm{m}$ band data of most sources. Moreover, the $3.6 \mu \mathrm{m}$ band is probing the continuum emission in a spectral regime where the power-law approximation may no longer be valid (particularly at high $M_{\mathrm{BH}}$; see, e.g., Davis \& Laor 2011; Netzer \& Trakhtenbrot 2014). In what follows, we focus on the $L_{4.5}$-based estimates of $\dot{M}_{\text {disk }}$, as these would result in more conservative constraints on $\eta$ (i.e., lower limits; see below).

We next use the estimates of $\dot{M}_{\text {disk }}$ and the observed luminosities of our quasars to investigate the range of - or lower limits on $-\eta$, which would be consistent with all the data available for the $z \simeq 6$ quasars, following $\eta=L_{\mathrm{bol}} / \dot{M}_{\mathrm{disk}} c^{2}$. Given the data in hand, $L_{\text {bol }}$ can only be estimated by utilizing bolometric corrections and monochromatic luminosities (unlike the analysis of Davis \& Laor 2011).

We focus on conservative, lower limits on $\eta$, which can be obtained assuming $L_{\mathrm{bol}}=3 \times L_{3.6}\left(\simeq 3 \times \lambda L_{\lambda}[4930 \AA]\right)$.

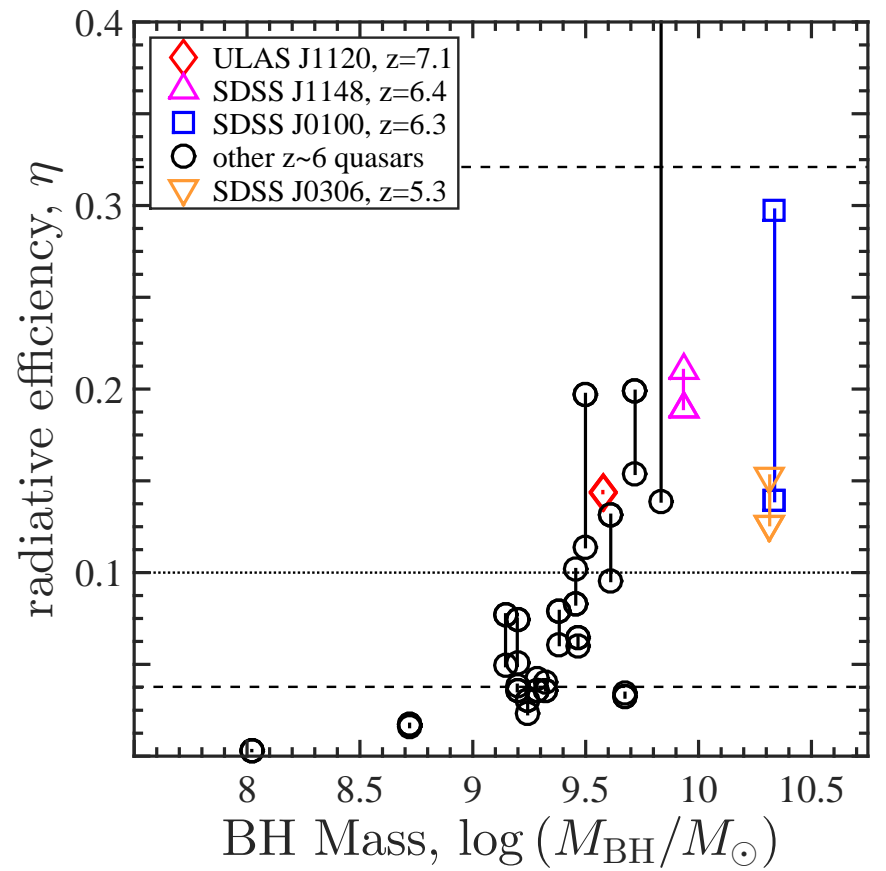

FIG. 3.- Estimates of radiative efficiencies $\eta$, vs. BH mass, $M_{\mathrm{BH}}$, for the 20 quasars compiled in our sample. For each quasar, we show two different estimates of $\eta$, with the lower value being our most conservative estimate (based on $L_{\text {bol }}=3 \times L_{3.6}$ and the $L_{4.5}$-based $\dot{M}_{\text {disk }}$ estimates), and the higher value derived through the $L_{3000}$-based $L_{\text {bol }}$ estimates (the TN12 bolometric corrections). The real $\eta$ may be higher than what these two sets of estimates suggest. The most massive BHs in our sample show high radiative efficiencies, $\eta \gtrsim 0.2$. The apparent trend of increasing $\eta$ with increasing $M_{\mathrm{BH}}$ is likely driven by the form of Equation 1. We cannot rule out that SMBHs with $M_{\mathrm{BH}} \gtrsim 10^{10} M_{\odot}$ and $\eta \lesssim 0.1$ exist, but are not (yet) observed.

This choice is very similar to the one made in Trakhtenbrot (2014) $\left(L_{\mathrm{bol}}=3 \times \lambda L_{\lambda}[5100 \AA]\right)$. We consider it to provide a lower limit on the real $L_{\text {bol }}$ since it reflects a bolometric correction that is much smaller, by at least a factor of $\sim 2$, than those used in many other studies of $M_{\mathrm{BH}}$ and $L / L_{\mathrm{Edd}}$ in high-redshift quasars (see, e.g., Runnoe et al. 2012). Since we seek to derive lower limits on $\eta$, we further use the higher, $L_{4.5}$-based estimates of $\dot{M}_{\text {disk }}$. As $\eta \propto 1 / \dot{M}_{\text {disk }} \propto M_{\mathrm{BH}}$, our $\eta$ estimates inherit the systematic uncertainties on $M_{\mathrm{BH}}$ (see above). Figure 2 shows the cumulative distribution of the conservative constraints on $\eta$ we obtain, which are in the range $\eta \gtrsim 0.003-0.2$. Most quasars have lower limits on $\eta$ that are consistent with what is expected for thin disks, and only three quasars have lower limits on $\eta$ that are below 0.03 . For the three quasars of particular interest we find conservative lower limits of $\eta \gtrsim 0.14,0.17$, and 0.2 (for ULAS J1120, SDSS J1148, and SDSS J0100, respectively). For SDSS J0306 we find $\eta \gtrsim 0.15$, consistent with the $z \simeq 6$ quasars and particularly with SDSS J0100, which has a very similar BH mass.

To further test the range of $\eta$ consistent with the data, we also calculated $L_{\text {bol }}$ using the $L_{3000}$-dependent bolometric corrections of $\mathrm{TN} 12$, which for the sample in hand are in the range $f_{\text {bol }}(3000 \AA) \sim 2-3.2$. Coupling these $L_{\text {bol }}$ estimates with the $L_{4.5}$-based $\dot{M}_{\text {disk }}$ estimates, we obtain $\eta$ estimates in the range of $\eta \simeq 0.003-0.44$ (see Table 1 and the dashed line in Figure 2). In this case, 12 of the 20 quasars $(60 \%)$ have $\eta$ within the range of values expected for thin disks. Only two objects have $\eta<0.03$, and only one has $\eta>0.3$. Focusing again on the three quasars mentioned above, we obtain 


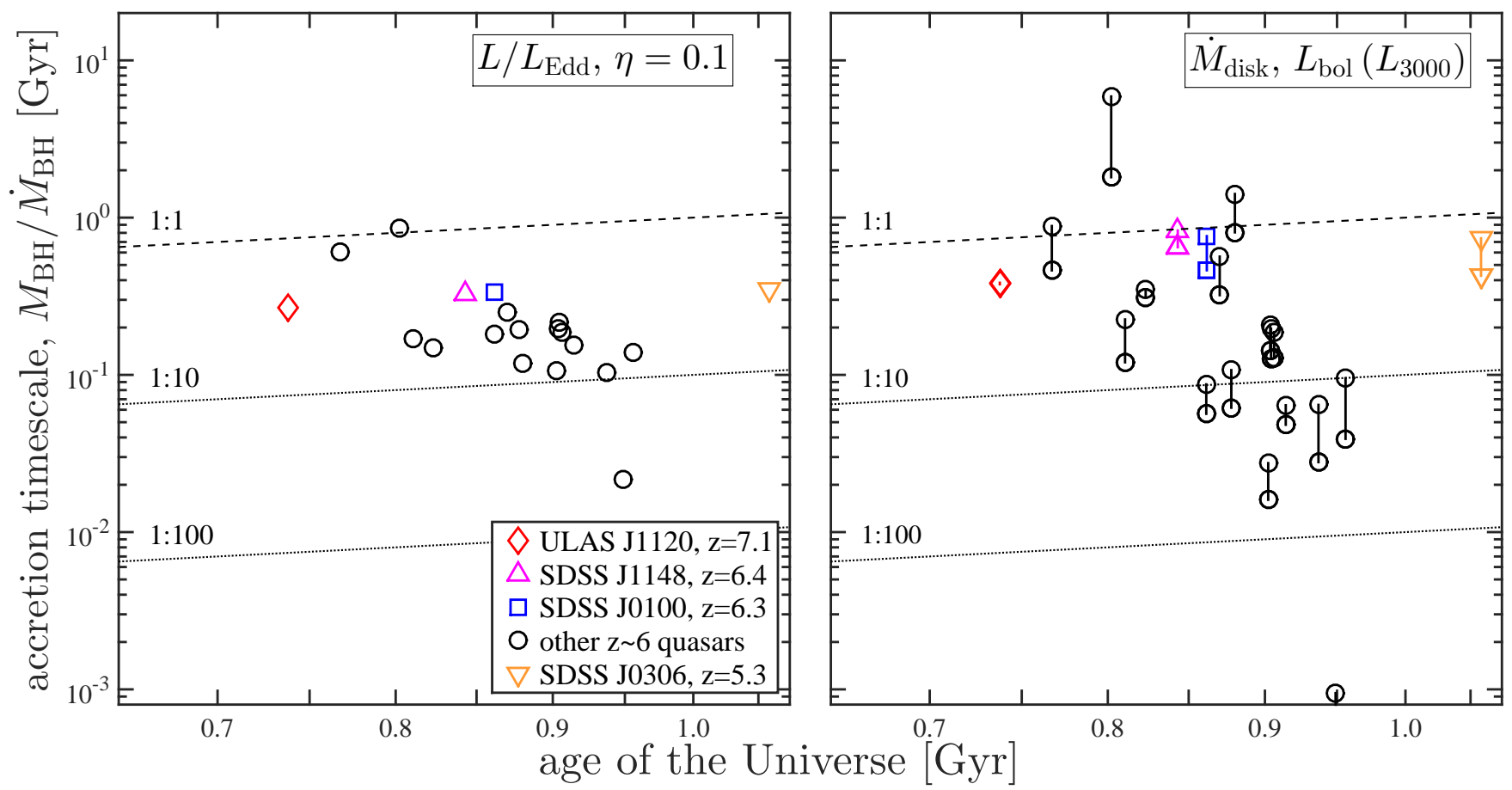

FIG. 4. - Different estimates of BH accretion timescales, $t_{\mathrm{acc}}=M_{\mathrm{BH}} / \dot{M}_{\mathrm{BH}}$, vs. cosmic epoch. Left: "standard" $t_{\mathrm{acc}}$ timescale estimates, obtained from $L / L_{\mathrm{Edd}}$ and assuming $\eta=0.1$. Right: $t_{\text {acc }}$ estimates obtained from $\dot{M}_{\text {disk }}$ and the $L_{3000}$-based estimates of $L_{\text {bol }}$ (i.e., equivalent to using our $\eta$ estimates; see Table 1 ). For each source, we plot the timescales obtained from both the $L_{3.6}$ - and $L_{4.5}$-based estimates of $\dot{M}_{\text {disk }}$.

$\eta \simeq 0.14,0.19$, and 0.14 , for ULAS J1120, SDSS J1148, and SDSS J0100, respectively. For SDSS J0306 at $z=5.36$ we find $\eta \simeq 0.13$, again highly consistent with SDSS J0100.

We note that these latter $L_{3000}$-based estimates of $L_{\text {bol }}$ and $\eta$ may also be considered conservative, as the TN12 bolometric corrections we use are, again, significantly lower than those commonly used for samples of high- $z$ quasars (by factors of $\sim 2$; see, e.g., the compilation of Runnoe et al. 2012). If we had instead used the $f_{\text {bol }}(3000 \AA)$ suggested by Runnoe et al. (2012), the resulting $\eta$ estimates would have been higher by $\sim 30 \%$.

Figure 3 presents our two sets of (conservative) $\eta$ estimates of $\eta$ against $M_{\mathrm{BH}}$. The apparent trend of increasing $\eta$ with increasing $M_{\mathrm{BH}}$ is mainly driven by the explicit dependence of Equation 1 on $M_{\mathrm{BH}}$, and then on the fact that $\eta \propto 1 / \dot{M}_{\text {disk }}$ (at fixed luminosity). It is therefore expected that rest-frame $\mathrm{UV}$-optical surveys of a given depth would miss high- $M_{\mathrm{BH}}$ but low- $\eta$ objects (see also Bertemes et al. 2016). In this context, extremely massive objects like SDSS J0100 and SDSS J0306, which have $M_{\mathrm{BH}} \gtrsim 10^{10} M_{\odot}$, may represent the high- $\eta$ end of a much larger population of high-mass BHs at $z \sim 5-6$. A large enough number of such extreme, yet-to-be-observed objects may further constrain models of $\mathrm{BH}$ seed formation and early growth (e.g., Agarwal et al. 2013).

We next turn to estimate the accretion timescales of the SMBHs powering the quasars, $t_{\mathrm{acc}} \equiv M_{\mathrm{BH}} / \dot{M}_{\mathrm{BH}}$, which may be considered as the typical $\mathrm{BH}$ mass $e$-folding timescales. Here, we derive and compare two sets of $t_{\text {acc }}$ estimates made available by the data, and following two approaches for estimating $\dot{M}_{\mathrm{BH}}=(1-\eta) \dot{M}_{\text {disk }}$. First, we follow the common procedure of using $L / L_{\text {Edd }}$ and a fixed radiative efficiency (i.e., $\eta=0.1$ ), to derive accretion timescales through $t_{\text {acc }} \simeq 0.4(\eta / 1-\eta)$ Gyr. The $t_{\text {acc }}$ estimates thus obtained are shown in the left panel of Figure 4, plotted against the age of the universe (at the observed epoch). Most objects have $t_{\text {acc }} \sim 0.1-1 \mathrm{Gyr}$, and could have had between $\sim 1-10$ mass $e$-foldings of $M_{\mathrm{BH}}$. Second, we use our $\dot{M}_{\text {disk }}$ estimates, which provide $\dot{M}_{\mathrm{BH}}=(1-\eta) \dot{M}_{\text {disk }}$, and our conservative estimates of $\eta$. Due to the dependence of $\eta$ on $\dot{M}_{\text {disk }}$ in our analysis, we note that these estimates can be expressed as $t_{\mathrm{acc}}=M_{\mathrm{BH}} /\left(\dot{M}_{\text {disk }}-L_{\mathrm{bol}} / c^{2}\right)$. As before, we adopt the $L_{3000^{-}}$

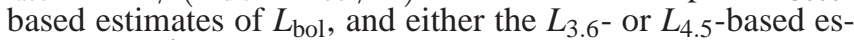
timates of $\dot{M}_{\text {disk }}$. The accretion timescales we obtain through this procedure are presented in the right panel of Figure 4. The shorter, $L_{3.6}$-based timescales, are generally in the range of $t_{\text {acc }} \sim 0.01-2 \mathrm{Gyr}$, with 18 of the quasars (90\%) having $t_{\text {acc }} \simeq 0.03-0.8$ Gyr. The extremely high- $\dot{M}_{\text {disk }}$ quasar J0005 $\left(\dot{M}_{\text {disk }}>100 M_{\odot} \mathrm{yr}^{-1}\right)$ has the shortest accretion timescale, $t_{\text {acc }} \simeq 0.5 \mathrm{Myr}$ (cf. $\sim 0.02$ Gyr obtained from $L / L_{\text {Edd }}$ ). For the three $z>6$ quasars of interest, ULAS J1120, SDSS J1148, and SDSS J0100, we find $t_{\mathrm{acc}}=0.36,0.71$ and $0.67 \mathrm{Gyr}$, respectively. The ultramassive $z \simeq 5.3$ quasar SDSS J0306 has $t_{\text {acc }}=0.66$ Gyr. The $\dot{M}_{\text {disk }}$-based estimates of $t_{\text {acc }}$ suggest that some quasars could have had as many as $50 \mathrm{BH}$ mass $e$-foldings.

We stress, again, that the trends of decreasing $t_{\mathrm{acc}}$ with epoch seen in Figure 4 are due to the way the original samples of quasars were selected and identified (i.e., their brightness and luminosity), and the form of the prescriptions we use here.

The two sets of $t_{\text {acc }}$ estimates are generally in good agreement, with differences being within a factor of $\sim 2$ for 14 (70\%) of our quasars, and even within a factor of $\sim 1.5$ for $8(40 \%)$ of the quasars. The lack of a significant systematic offset between the two timescale estimates reflects the fact that our $\eta$ estimates bracket the "standard" value of $\eta=0.1$. We conclude that for our sample of $z \simeq 6$ quasars, the simpler $L / L_{\text {Edd }}$-based growth timescale estimates are broadly consistent with those derived through our more elaborate approach.

\section{DISCUSSION AND CONCLUSION}


The main point of our analysis is to investigate whether the data available for some of the highest-redshift quasars known to date can be accounted for, self-consistently, within the generic model of a radiatively efficient, thin accretion disk.

We found that the accretion rates through the postulated thin disks are generally in the range of $\dot{M}_{\text {disk }} \sim 1-$ $100 M_{\odot} \mathrm{yr}^{-1}$. These accretion rates are consistent with the systems being Eddington-limited, at the observed epoch. However, if one assumes that these accretion rates were sustained at earlier epochs, when the $\mathrm{BH}$ masses were considerably lower, this would imply super-Eddington accretion rates, which may be sustained under certain gas configurations, and lead to a fast buildup of BH mass (e.g., Volonteri et al. 2015).

We showed that the available data for most of the $z \simeq 6$ quasars can be explained with conservative estimates (lower limits) on the radiative efficiencies that are in the range $0.04 \lesssim$ $\eta \lesssim 0.32$ - that is, within the range expected for accretion through a thin disk onto rotating BHs. Our more conservative estimates suggest $\eta \gtrsim 0.05$ for most objects. Thus, it appears that all the data available for quasars at $z \gtrsim 5.8$ are consistent with such radiatively efficient accretion flows. Moreover, since our analysis provides lower limits on $\eta$, it is possible that the real $\eta$ of the quasars under study would differ substantially with the expectations of radiatively inefficient accretion flow models.

We stress that this result is not a trivial consequence of the observables and methodology we adopt here (i.e., Equation 1) . For example, the study of Trakhtenbrot (2014) - applying the same methods as the ones used here to a sample of high$M_{\mathrm{BH}}$ quasars at $1.5 \lesssim z \lesssim 3.5$ - found extremely high values of $\eta$, which in many cases exceeded $\eta \simeq 0.4$. Among the conservative $\eta$ estimates, we find $\eta \gtrsim 0.15$ for ULAS J1120 - the highest-redshift quasar known to date $(z=7.1)$, and $\eta \gtrsim 0.2$ for SDSS J1148 (at $z=6.4$ ). On the other hand, many other $z \simeq 6$ quasars have $0.05 \lesssim \eta \lesssim 0.1$ - below the standard, universal radiative efficiency assumed in many studies of the AGN population. The limited size of our sample prevents us from determining whether these $\eta$ estimates represent the scatter within the quasar population, or only trace a few extreme cases.

The accretion timescales of the SMBHs under study, derived assuming the $\dot{M}_{\text {disk }}$ and $\eta$ estimates, are consistent with those derived from $L / L_{\mathrm{Edd}}$ and the universal $\eta=0.1$ assumption, allowing for $\sim 1-100$ mass $e$-foldings. This further justifies the usage of the simpler $t_{\mathrm{acc}}$ estimates in cases where only $L / L_{\text {Edd }}$ is available (i.e., when rest-frame optical luminosities are unavailable). However, this assumption would naturally neglect the fact that any population of accreting SMBHs is expected to have a range of $\eta$.

Within the standard thin-disk framework, radiative efficiencies are closely linked to $\mathrm{BH}$ spin, $a_{*}$ (in normalized units). The range of $\eta$ we find corresponds to the entire possible range of $-1 \leq a_{*} \leq 1$, and the typical (median) $\eta$ of our sample corresponds to $a_{*} \simeq 0.7$ - again consistent with what is expected from the assumption of a universal $\eta=0.1$. As the quasars of interest in our sample (ULAS J1120, SDSS J1148, and SDSS J0100) have $\eta>0.1$, they correspond to rather high spins, $a_{*} \gtrsim 0.9$. These, in turn, are consistent with what is found for low-redshift, lowluminosity AGNs (Reynolds 2014, and references therein) and for higher-luminosity, higher- $M_{\mathrm{BH}}$ quasars at higher redshifts (Reis et al. 2014; Trakhtenbrot 2014; Capellupo et al. 2016). This supports a scenario in which the $z \simeq 6 \mathrm{SMBHs}$ grew through coherent accretion flows (e.g., Dotti et al. 2013; Volonteri et al. 2013, and references therein). This "spin up" scenario appears highly plausible, given the high duty cycle of accretion required for the fast $\mathrm{BH}$ growth at $z>6$.

Several recent studies highlighted the possibility that $z \simeq$ 6 quasars could have grown through "super-critical" accretion, to reach their high BH masses (Volonteri \& Rees 2005; Alexander \& Natarajan 2014; Volonteri et al. 2015). The more extreme cases of such accretion, in slim disks, may result in $\dot{m} / \dot{m}_{\text {Edd }} \sim 100$ and $\eta \sim 0.01$, essentially regardless of the $\mathrm{BH}$ spin (see, e.g., Sadowski 2009; Madau et al. 2014; McKinney et al. 2014; Volonteri et al. 2015, but see McKinney et al. 2015). Our analysis confirms that such super-critical growth episodes should have occurred, if at all, in the yet earlier universe, when BHs were smaller, to alleviate the requirement of continuous growth, which may not be realistic.

The data currently available for the highest redshift quasars, namely, in the (rest-frame) UV and optical, as well as the data that may become available in the foreseeable future, cannot directly distinguish between radiatively efficient and inefficient accretion. Models of such flows require further study, with an emphasis on the observables that are relevant for faint, $z>6$ sources. For instance, super-critical episodes are likely accompanied by the production of powerful jets (McKinney et al. 2014; Sadowski et al. 2014; Sadowski \& Narayan 2016). At high- $z$ such jets should be detectable in X-rays rather than as extended radio sources (Ghisellini et al. 2015). This, together with the limitations present in the (rest-frame) UV regime due to IGM absorption, highlight the importance of the $\mathrm{X}$-ray regime, where surveys of ever-increasing depth (e.g., in the CDF-S field) may provide key insights into the assembly of the earliest SMBHs.

We appreciate the feedback from the anonymous referee, which helped us to improve the manuscript. We thank K. Schawinski for beneficial discussions. This work was performed in part at the Aspen Center for Physics, which is supported by National Science Foundation grant PHY1066293. M.V. acknowledges funding from the European Research Council under the European Community's Seventh Framework Programme (FP7/2007-2013 Grant Agreement No. 614199, project 'BLACK'). P.N. acknowledges support from TCAN grant with award number 1332858 from the National Science Foundation. This work made use of the MATLAB package for astronomy and astrophysics (Ofek 2014). 


\section{REFERENCES}

Agarwal, B., Davis, A. J., Khochfar, S., Natarajan, P., \& Dunlop, J. S. 2013, MNRAS, 432, 3438 [3]

Alexander, T., \& Natarajan, P. 2014, Science, 345, 1330 [4]

Barnett, R., Warren, S. J., Banerji M., et al. 2015, A\&A, 575, A31 [2, 1]

Barth, A. J., Martini, P., Nelson, C. H., \& Ho, L. C. 2003, ApJL, 594, L95 [2]

Bertemes, C., Trakhtenbrot, B., Schawinski, K., Done, C., \& Elvis, M. 2016, MNRAS, 463, 4041 [3]

Bian, W., \& Zhao, Y.-H. 2003, PASJ, 55, 599 [2]

Capellupo, D. M., Netzer, H., Lira, P., Trakhtenbrot, B., \& Mejía-Restrepo, J. E. 2016, MNRAS, 460, $212[1,4]$

Davis, S. W., \& Laor, A. 2011, ApJ, 728, 98 [1, 2, 2, 3, 3]

De Rosa, G., Decarli, R., Walter, F., Fan, X., Jiang, L., Kurk, J. D., Pasquali, A., \& Rix, H. 2011, ApJ, 739, $56[1,2,1]$

De Rosa, G., Venemans, B. P., Decarli, R., et al. 2014, ApJ, 790, 145 [1, 2, 1]

Dotti, M., Colpi, M., Pallini, S., Perego, A., \& Volonteri, M. 2013, ApJ, 762, 68 [4]

Ghisellini, G., Haardt, F., Ciardi, B., Sbarrato, T., Gallo, E., Tavecchio, F., \& Celotti, A. 2015, MNRAS, 452, 3457 [4]

Habouzit, M., Volonteri, M., \& Dubois, Y. 2016, arXiv:1605.09394 [1]

Iwamuro, F., Kimura, M., Eto, S., Maihara, T., Motohara, K., Yoshii, Y., \& Doi, M. 2004, ApJ, 614, 69 [2]

Jarrett, T. H., Cohen, M., Masci, F., et al. 2011, ApJ, 735, 112 [2]

Jiang, L., Fan, X., Vestergaard, M., Kurk, J. D., Walter, F., Kelly, B. C., \& Strauss, M. A. 2007, AJ, 134, 1150 [2]

Jiang, L., Fan, X., Hines, D. C., et al. 2006, AJ, 132, 2127 [2, 1]

Kurk, J. D., Walter, F., Fan, X., Jiang, L., Jester, S., Rix, H., \& Riechers, D. a. 2009, ApJ, 702, 833 [2]

Kurk, J. D., Walter, F., Fan, X., et al. 2007, ApJ, 669, 32 [1, 2, 1]

Leipski, C., Meisenheimer, K., Walter, F., et al. 2014, ApJ, 785, 154 [2, 1]

Madau, P., Haardt, F., \& Dotti, M. 2014, ApJL, 784, L38 [4]

McKinney, J. C., Dai, L., \& Avara, M. J. 2015, MNRAS Letters, 454, L6 [4]

McKinney, J. C., Tchekhovskoy, A., Sadowski, A., \& Narayan, R. 2014, MNRAS, 441, 3177 [4]

Mortlock, D. J., Warren, S. J., Venemans, B. P., et al. 2011, Nature, 474, 616 [2]
Narayan, R., \& Yi, I. 1995, ApJ, 452, 710 [1]

Netzer, H., \& Trakhtenbrot, B. 2014, MNRAS, 438, 672 [2, 3]

Ofek, E. O. 2014, MATLAB package for astronomy and astrophysics, Astrophysics Source Code Library, ascl: 1407.005 [4]

Paczyńsky, B., \& Wiita, P. J. 1980, A\&A, 88, 23 [1]

Reis, R. C., Reynolds, M. T., Miller, J. M., \& Walton, D. J. 2014, Nature 507, 207 [4]

Reynolds, C. S. 2014, SSRv, 183, 277 [1, 4]

Runnoe, J. C., Brotherton, M. S., \& Shang, Z. 2012, MNRAS, 422, 478 [3]

Sadowski, A. 2009, ApJS, 183, 171 [4]

Sadowski, A., \& Narayan, R. 2016, MNRAS, 456, 3929 [4]

Sadowski, A., Narayan, R., Mckinney, J. C., \& Tchekhovskoy, A. 2014, MNRAS, 439, 503 [4]

Shen, Y., Richards, G. T., Strauss, M. A., et al. 2011, ApJS, 194, 45 [2]

Trakhtenbrot, B. 2014, ApJL, 789, L9 [1, 2, 3, 4]

Trakhtenbrot, B., \& Netzer, H. 2012, MNRAS, 427, 3081

Trakhtenbrot, B., Netzer, H., Lira, P., \& Shemmer, O. 2011, ApJ, 730, 7 [1]

Trakhtenbrot, B., Civano, F., Urry, C. M., et al. 2016, ApJ, 825, 4 [1]

Treister, E., Schawinski, K., Volonteri, M., \& Natarajan, P. 2013, ApJ, 778 $130[1]$

Venemans, B. P., Findlay, J. R., Sutherland, W. J., et al. 2013, ApJ, 779, 24 [2]

Venemans, B. P., Bañados, E., Decarli, R., et al. 2015, ApJL, 801, L11 [2, 1]

Volonteri, M., \& Rees, M. J. 2005, ApJ, 633, 624 [4]

Volonteri, M., \& Reines, A. 2016, ApJL, 820, L6 [1]

Volonteri, M., Sikora, M., Lasota, J.-P., \& Merloni, A. 2013, ApJ, 775, 94 [4]

Volonteri, M., Silk, J., \& Dubus, G. 2015, ApJ, 804, 148 [4]

Wang, F., Wu, X.-B., Fan, X., et al. 2015, ApJL, 807, L9 [2, 1]

Willott, C. J., Albert, L., Arzoumanian, D., et al. 2010, AJ, 140, 546 [1, 2, 1]

Wright, E. L., Eisenhardt, P. R. M., Mainzer, A. K., et al. 2010, AJ, 140, 1868 [2]

Wu, S., Lu, Y., \& Zhang, F. 2013, MNRAS, 436, 3271 [2]

Wu, X.-B., Wang, F., Fan, X., et al. 2015, Nature, 518, 512 [2, 1] 
TABLE 1

OBSERVED AND DERIVED PROPERTIES

\begin{tabular}{|c|c|c|c|c|c|c|c|c|c|c|}
\hline Object & $z^{\mathrm{a}}$ & $\begin{array}{c}\log L_{3000}{ }^{\mathrm{b}} \\
\left(\mathrm{erg} \mathrm{s}^{-1}\right)\end{array}$ & $\begin{array}{c}\text { FWHM(Mg II) } \\
\left(\mathrm{km} \mathrm{s}^{-1}\right)\end{array}$ & $\begin{array}{l}\text { NIR } \\
\text { Ref. }^{c}\end{array}$ & $\begin{array}{c}\log M_{\mathrm{BH}}{ }^{\mathrm{d}} \\
\left(M_{\odot}\right)\end{array}$ & $\begin{array}{l}\log L_{3.6}{ }^{\mathrm{e}} \\
\left(\operatorname{erg~s}{ }^{-1}\right)\end{array}$ & $\begin{array}{l}\log L_{4.5}{ }^{\mathrm{e}} \\
\left(\operatorname{erg~s}^{-1}\right)\end{array}$ & $\begin{array}{l}\text { MIR } \\
\text { Ref. }^{f}\end{array}$ & $\begin{array}{c}\dot{M}_{\text {disk }}{ }^{\mathrm{g}} \\
\left(M_{\odot} \mathrm{yr}^{-1}\right)\end{array}$ & $\eta^{\mathrm{h}}$ \\
\hline $\mathrm{J} 1120+0641$ & 7.097 & 46.48 & 4411 & 2 & 9.58 & 46.50 & 46.36 & 3 & 11.4 & 0.144 \\
\hline $\mathrm{J} 1148+5251$ & 6.407 & 46.79 & 5352 & 1 & 9.93 & 46.73 & 46.65 & 1 & 16.3 & 0.189 \\
\hline $\mathrm{J} 0100+2802$ & 6.3 & 47.50 & 5130 & 4 & 10.34 & 47.31 & 47.24 & 2 & 54.6 & 0.139 \\
\hline $\mathrm{J} 0306+1853$ & 5.363 & 47.31 & 5722 & 5 & 10.32 & 47.20 & 47.15 & 2 & 55.4 & 0.126 \\
\hline $\mathrm{J} 0050+3445$ & 6.253 & 46.55 & 4360 & 6 & 9.61 & 46.42 & 46.37 & 2 & 14.6 & 0.131 \\
\hline $\mathrm{J} 0836+0054$ & 5.81 & 46.93 & 3600 & 7 & 9.67 & 46.91 & 47.01 & 1 & 124.4 & 0.032 \\
\hline $\mathrm{J} 0353+0104$ & 6.072 & 46.42 & 3682 & 1 & 9.38 & 46.32 & 46.29 & 1 & 18.4 & 0.079 \\
\hline $\mathrm{J} 0842+1218$ & 6.069 & 46.47 & 3931 & 1 & 9.47 & 46.45 & 46.43 & 1 & 24.6 & 0.065 \\
\hline $\mathrm{J} 2348-3054$ & 6.889 & 45.99 & 5446 & 2 & 9.46 & 46.11 & 46.10 & 2 & 6.7 & 0.083 \\
\hline J0305-3150 & 6.605 & 46.24 & 3189 & 2 & 9.15 & 46.07 & 46.05 & 2 & 12.6 & 0.077 \\
\hline $\mathrm{P} 036+03$ & 6.527 & 46.68 & 3500 & 3 & 9.50 & 46.44 & 46.28 & 2 & 12.7 & 0.197 \\
\hline $\mathrm{P} 338+29$ & 6.658 & 46.11 & 6800 & 3 & 9.72 & 46.02 & 46.07 & 2 & 3.6 & 0.199 \\
\hline J0005-0006 & 5.844 & 46.00 & 1036 & 1 & 8.02 & 46.02 & 46.03 & 1 & 186.6 & 0.003 \\
\hline $\mathrm{J} 1411+1217$ & 5.903 & 46.57 & 2824 & 1 & 9.24 & 46.45 & 46.55 & 1 & 64.8 & 0.031 \\
\hline $\mathrm{J} 1306+0356$ & 6.017 & 46.34 & 3158 & 1 & 9.20 & 46.39 & 46.34 & 1 & 33.9 & 0.036 \\
\hline $\mathrm{J} 1630+4012$ & 6.058 & 46.25 & 3366 & 1 & 9.20 & 46.11 & 46.07 & 1 & 13.3 & 0.075 \\
\hline J0303-0019 & 6.079 & 46.01 & 2307 & 1 & 8.72 & 46.00 & 46.02 & 1 & 33.3 & 0.017 \\
\hline $\mathrm{J} 1623+3112$ & 6.211 & 46.36 & 3587 & 1 & 9.32 & 46.43 & 46.45 & 1 & 35.2 & 0.036 \\
\hline $\mathrm{J} 1048+4637$ & 6.198 & 47.28 & 3366 & 1 & 9.83 & 46.60 & 46.54 & 1 & 15.2 & 0.444 \\
\hline $\mathrm{J} 1030+0524$ & 6.302 & 46.36 & 3449 & 1 & 9.28 & 46.45 & 46.43 & 1 & 35.2 & 0.036 \\
\hline
\end{tabular}

a Redshift measured from the best-fit model of the Mg II line.

${ }^{\mathrm{b}}$ Monochromatic luminosity $\left(\lambda L_{\lambda}\right)$ at rest-frame wavelength of $3000 \AA$, obtained from the best-fit model of the Mg II emission line complex.

${ }^{c}$ References for NIR spectral analysis and Mg II measurements: (1) De Rosa et al. (2011); (2) De Rosa et al. (2014); (3) Venemans et al. (2015); (4) Wu et al. (2015); (5) Wang et al. (2015); (6) Willott et al. (2010); (7) Kurk et al. (2007).

${ }^{\mathrm{d}} \mathrm{BH}$ mass, estimated using the $\mathrm{Mg}$ II line and the TN12 prescription.

${ }^{\text {e }}$ Monochromatic luminosities $\left(\lambda L_{\lambda}\right)$ at observed-frame wavelengths of $\sim 3.6$ and $4.5 \mu \mathrm{m}$.

${ }^{\mathrm{f}}$ References for MIR photometry: (1) Spitzer (Leipski et al. 2014; including detections by Jiang et al. 2006); (2) WISE cross-match (see also Wu et al. 2015 for J0100 and Wang et al. 2015 for J0306); (3) Spitzer (Barnett et al. 2015).

g Obtained using Equation 1, $L_{4.5}$ and $M_{\mathrm{BH}}$.

${ }^{\text {h }}$ Obtained using the bolometric corrections of TN12, and the $L_{4.5}$-based $\dot{M}_{\text {disk }}$. 